\section{Prostatakarzinom: Bestrahlung könnte zusätzliche Vorteile bringen}

\begin{abstract}
Über die optimale Behandlung von Patienten mit lokal fortgeschrittenem Prostatakarzinom wird derzeit noch kontrovers diskutiert. Einer aktuellen Studie zufolge könnten Patienten, die eine antiandrogene Behandlung erhalten, von einer zusätzlichen Radiotherapie profitieren.
\end{abstract}

\begin{abstract}
n einer internationalen Studie wurde untersucht, ob Prostatakarzinompatienten durch eine zusätzliche Radiotherapie (RT) zur Androgendeprivationstherapie (ADT) einen Überlebensvorteil haben. Die Patienten hatten ein lokal fortgeschrittenes Karzinom (T3 oder T4) oder eine organbeschränkte (T2) Erkrankung, mit einem PSA-Wert über $40 \mathrm{ng} / \mathrm{ml}$ oder über $20 \mathrm{ng} / \mathrm{ml}$ und einem Gleason-Score von mindestens 8 . Sie erhielten entweder eine ADT allein oder zusätzlich eine RT (65-69 Gy auf Prostata und Samenbläschen, 45 Gy auf die Beckenlymphknoten). 602 Patienten wurden einer reinen ADT
\end{abstract}

und 603 einer kombinierten Behandlung mit RT zugeordnet.

Zum Zeitpunkt der zweiten Zwischenauswertung Ende 2008 waren in der ADTGruppe 175 und in der ADT+RT-Gruppe 145 Patienten verstorben. So hatte die zusätzliche RT einen Gesamtüberlebensvorteil von $23 \%$ gebracht (HR 0,77; p = 0,03). Das Sieben-Jahres-Gesamtüberleben lag in der ADT-Gruppe bei $66 \%$, unter zusätzlicher RT bei 74\%. Während in der ADTGruppe $51 \%$ ihrem Karzinom zum Opfer fielen, verstarben in der Kombinationsgruppe nur $35 \%$ infolge des Krebses. Damit reduzierte die zusätzliche RT die krebs- bedingte Sterberate um $46 \%$ (HR 0,54; p $=0,0001)$. Das krankheitsspezifische Sieben-Jahres-Überleben lag in der ADTGruppe bei $79 \%$, bei zusätzlicher RT erreichte es $90 \%$. Zudem zeichnete sich ab, dass eine zusätzliche RT das Fortschreiten der Erkrankung deutlich verzögern kann.

Fazit: Den Autoren zufolge sollte die Möglichkeit einer kombinierten Behandlung aus ADT und RT mit allen Patienten mit lokal fortgeschrittenem Prostatakarzinom besprochen werden. Eine alleinige ADT für Männer mit Hochrisiko-Prostatakarzinomen sei nicht angemessen. Ob jedoch die optimale Ersttherapie eine Kombination aus ADT plus RT sei oder aber eine Operation gefolgt von einer selektiven RT sei nach wie vor offen. Dr. Christine Starostzik

Warde $P$ et al. Combined androgendeprivation therapy and radiation therapy for locally advanced prostate cancer: a randomised, phase 3 trial. Lancet 2012; 378: 2104-11

\title{
Laser oder TURP - was ist besser bei BPH?
}

\section{Bei benigner Prostatahyperplasie mit dem Holmium-Laser oder mit der Elektroschlinge aushöhlen? Neuseeländische Forscher haben beide Methoden untersucht und nun aktuelle Sieben-Jahres-Daten veröffentlicht.}

Z ur Entfernung von Prostatagewebe bei benigner Prostatahyperplasie (BPH) sind sowohl die Holmiumlaserenukleation (HoLEP) als auch die transurethrale Re-

\begin{tabular}{|l|l|l|}
\hline \multicolumn{3}{|l|}{$\begin{array}{l}\text { Vergleich verschiedener } \\
\text { Parameter nach HoLEP oder TURP }\end{array}$} \\
\hline & HoLEP & TURP \\
\hline Harnflussrate $Q_{\max }$ (mI/s) & 22,09 & 17,83 \\
\hline $\begin{array}{l}\text { American Urological Associati- } \\
\text { on-(AUA-)Symptom-Score }\end{array}$ & 8,0 & 10,3 \\
\hline $\begin{array}{l}\text { Quality of Life Index } \\
\text { (QoL-Score) }\end{array}$ & 1,47 & 1,31 \\
\hline $\begin{array}{l}\text { Benign Prostatic Hyperplasia- } \\
\text { Impact-Index (BPH-II) }\end{array}$ & 1,53 & 0,58 \\
\hline $\begin{array}{l}\text { International Index } \\
\text { of Erectile Function (IIEF) }\end{array}$ & 11,6 & 9,21 \\
\hline \begin{tabular}{l} 
ICSmale Voiding Score (VS) \\
\hline \begin{tabular}{l} 
ICSmale Incontinence Score (IS) \\
\hline
\end{tabular}
\end{tabular} & 3,07 & 1,17 \\
\hline
\end{tabular}

sektion der Prostata (TURP) anerkannt, doch fehlten bisher vergleichende Langzeitdaten. Nachdem 2006 die Zwei-JahresErgebnisse publiziert worden waren, legten neuseeländische Autoren jetzt den Datenvergleich von HoLEP und monopolarer TURP nach sieben Jahren vor.

Jenseits des ersten Jahres nach dem Eingriff zeigte sich bei keinem der untersuchten Parameter ein signifikanter Unterschied zwischen den beiden Methoden. Allerdings ergab sich eine unterschiedliche Erfolgsrate bei der Notwendigkeit einer Nachbehandlung: Während keiner der BPH-Patienten nach der Lasertherapie eine zweite Operation benötigte, mussten sich drei der 17 Patienten nach TURP einer nochmaligen Behandlung wegen eines Rezidivs unterziehen. Nach einer mittleren Beobachtungszeit von 6,7 Jahren zeigten sich durchschnittlich die in der Tabelle zusammengefassten Ergebnisse. Die behandelten Patienten litten unter einer beeinflussten Urodynamik $\left(\mathrm{Q}_{\max }\right.$ $\leq 15 \mathrm{ml} / \mathrm{s}$ ), und hatten Prostatavolumina zwischen $40 \mathrm{ml}$ und $200 \mathrm{ml}$. Von den ursprünglich 61 Patienten konnten die Daten von 31 am Studienende im Durchschnitt rund 80 -jährigen Patienten (14 HoLEP, 17 TURP) vollständig ausgewertet werden. Perioperativ hatten sich für die HoLEP-Methode signfikante Vorteile hinsichtlich der Menge des entfernten Gewebes, der Katheterzeit und der Länge des Krankenhausaufenthalts ergeben. $6,7 \%$ der Patienten benötigten nach HoLEP postoperative Blasenspülungen im Vergleich zu $70 \%$ in der TURP-Gruppe.

Fazit: Die Ergebnisse der Laserbehandlung zeigten sich gegenüber der TURP als mindestens gleichwertig. Langfristig sind demnach nach HoLEP weniger Zweitoperationen notwendig. Um die Ergebnisse zu bestätigen sind jedoch multizentrische Studien mit wesentlich mehr Patieten notwendig. Dr. Christine Starostzik

Gilling PJ et al. Long-term results of a randomized trial comparing holmium laser enucleation of the prostate and transurethral resection of the prostate: results at 7 years. BJU Int 2011; 109: 408-11 